\title{
The Taipei Adolescent Twin/Sibling Family Study II: Depression, Insulin Resistance, and Hormonal Factors
}

\author{
Shu-Yu Kuo,' Pi-Hua Liu,' Lee-Ming Chuang,' and Wei J. Chen ${ }^{1,3}$ \\ ' Institute of Epidemiology, College of Public Health, National Taiwan University, Taipei, Taiwan \\ ${ }^{2}$ Department of Internal Medicine, College of Medicine and National Taiwan University Hospital, National Taiwan University, Taipei, Taiwan \\ ${ }^{3}$ Department of Psychiatry, College of Medicine and National Taiwan University Hospital, National Taiwan University, Taipei, Taiwan
}

In this ongoing longitudinal study of adolescent twins/sibling pairs and their parents in Taipei, we aimed to investigate the genetic and environmental influences on adolescent behavioral development, metabolic risk factors, and the associations between the two. Special focus is on anxious depression, metabolic profile, and hormonal factors such as cortisol and leptin. The first wave of assessment was completed during the period of 2002 to 2005 and included 192 twin pairs, 6 triplets, 56 sibling pairs and their first-degree relatives (484 parents and 142 siblings). We are currently in the process of a second wave assessment as follow-up. Dimensional psychological assessments using self-report questionnaires, as well as categorical assessments based on semistructured clinical interviews, were performed. All subjects received a 2-hour oral glucose tolerance test at the examination site. The metabolic phenotypes including body mass index, blood pressure, levels of glucose, insulin, and lipid profile as well as related hormonal levels were measured. Zygosity was determined using DNA, except for a few twins whose DNA was not available. Such a combination of detailed psychological assessments and metabolic function tests is expected to help shed light on the interrelation of psychological well-being and metabolic functioning.

A growing body of literature suggests that metabolic risk factors and anxious depression are closely interrelated. Differences in metabolic function indexes such as body mass index (BMI), levels of glucose, triglyceride, cholesterol, as well as blood pressure were observed in individuals with anxiety, depression, or both, in comparison to controls (Golden et al., 2004; McCaffery et al., 2003; Raikkonen et al., 2002). Intriguingly, these metabolic risk factors also tend to be clustered in adolescents (Cook et al., 2003; Weiss et al., 2004). Similarly, patients with major depression exhibited insulin resistance during an oral glucose tolerance test compared to controls (Winokur et al., 1988). A recent review concluded the existence of an association between depression and type 2 diabetes (Musselman et al., 2003). Furthermore, it has been shown that several hormones such as cortisol, an index of hypothalamic-pituitary-adrenal (HPA) axis activity and leptin, an adiposity hormone involved in food intake and weight regulation, have been involved in the regulation of mood and metabolic function (Atmaca et al., 2005; Bjorntorp, 2001; Kraus et al., 2001; Liuzzi et al., 1999; Livshits et al., 2005). Despite the consistent overlap between these two traits, few studies have adopted an integrative strategy that includes both psychological and physiological assessments to elucidate the etiology of anxiety/depression and metabolic functions.

Recognizing the need for integrating psychological and physiological information, we initiated a longitudinal, multivariate study of adolescent twins/sibling pairs aged 12 to 18 years, and their siblings and parents as well, in an attempt to investigate how psychological factors combine with biological changes to influence anxiety/depression and metabolic profiles. The design of this study allows us to assess the shared etiology underlying anxiety/depression, metabolic profile, and hormonal factors during adolescence and before the development of chronic illness. In particular, the study of correlated phenotypes with additional information from nontwin siblings and parents of twin probands would increase statistical power (Schmitz et al., 1998) given that the sample size was limited due to the comprehensive assessment required for this study.

\section{$\overline{\text { Recruitment Process }}$}

At baseline, participants were twin pairs or sibling pairs recruited from the junior high schools in Taipei during the period 2002 to 2005 in a study denoted as the Twin/Sibling Study of Insulin Resistance. We solicited a list of twins from 84 junior high schools in

Received 16 August, 2006; accepted 6 September, 2006.

Address for correspondence: Wei J. Chen, Institute of Epidemiology, College of Public Health, National Taiwan University, $17 \mathrm{Xu}$-Zhou Road, Taipei 100,Taiwan.E-mail: weijen@ha.mc.ntu.edu.tw 
Taipei City and County. Among them, 20 schools were unable to provide names of twins, and another four schools only provided the names of seven twin pairs who were willing to attend this study (without the number of potential twin pairs in their schools). Among those twins where names were available and were invited ( $n=1006$ families), 185 twin and six triplet families agreed to participate, yielding a family-wise participation rate of $19 \%$. The relatively low participation rate was probably owing to the time-consuming nature of the oral glucose tolerance test, which lasts 120 minutes with an indwelling venous line.

As monozygotic (MZ) twins are more common than dizygotic (DZ) twins in Taiwan (Chen et al., 1987), sibling pair families ( $n=56$; age variation within 2 years) from seven schools were also recruited to complement the number of DZ twins (Chen et al., 1999). In this study, all twins and sibling pairs were born and reared together in the same household. Participating subjects were unaware of specific hypotheses studied.

A total of 192 twin pairs, six triplets, 56 sibling pairs and their first-degree relatives (484 parents and 142 siblings) were included in the first wave of assessment from 2002 to 2005. The second wave of assessment commenced in July 2005 for the follow-up of subjects in a project denoted as Hormones and Metabolic Profiles in Relation to Adolescent Depression.

\section{Determination of Zygosity}

Twin zygosity was determined using DNA genotyping with five microsatellite DNA markers. The zygosity of five twin pairs, however, was classified based on the data of similarity questionnaire since their DNA samples were not available. The accuracy rate of zygosity determination using physical similarity data was estimated to be between 95.6 and $97.4 \%$ (Chen et al., 1999). The study was approved by the institutional review board of National Taiwan University Hospital. Informed written consent was obtained from all participating adolescents and their parents before their participation.

\section{Measurements}

The participating twins/siblings and their parents were asked to attend the examination center on the same date if possible. Self-report questionnaires and semistructural psychiatric interviews as well as physical assessments were completed at the examination site.

\section{Psychological Assessments}

At each wave of assessment, information on lifestyle including demographic, pubertal development, personality, substance use and abuse, and exercise as well as behavioral problems of twins/siblings and their parents were collected. Parents were also asked to fill out questionnaires, such as the Child Behavior Checklist (Achenbach, 1991; Yang et al., 2000), on the twins. For the second wave, categorical assessments were administered based on a semistructural clinical interview: the Chinese version of the Schedule for Affective Disorders and Schizophrenia for SchoolAge Children - Epidemiologic Version (K-SADS-E; Puig-Antich \& Orvaschel, 1990; Yang et al., 2004) for twins, and the Diagnostic Interview for Genetic Studies (DIGS; Chen et al., 1998; Nurnberger et al., 1994) for parents of twins. Table 1 lists the measures of behavioral problems, personality, and psychopathology that were collected in each wave of assessment.

\section{Physiological Assessments}

After an overnight fast ( $>8$ hours), baseline fasting blood samples were drawn for the measurement of levels of glucose and insulin, and lipids profile with the use of an indwelling venous line. The oral glucose tolerance test was then performed after subjects ingested $1.5 \mathrm{~g}$ glucose $/ \mathrm{kg}$ body weight up to a maximum of $75 \mathrm{~g}$ glucose solution. Blood samples were obtained for the analysis of plasma glucose and

\begin{tabular}{|c|c|c|}
\hline Psychological Measurements Used in the Taipei Adolescents Twin/Sibling Study & Wave 1 (2002) & Wave 2 (2005) \\
\hline \multicolumn{3}{|l|}{ For twins and siblings } \\
\hline Centers for Epidemiological Studies — Depression scale (CES-D) & $\mathrm{x}$ & $\mathrm{x}$ \\
\hline Tridimensional Personality Questionnaire, short form (TPQ) & - & $\mathrm{x}$ \\
\hline Youth Self-Report (YSR) & $\mathrm{x}$ & $\mathrm{x}$ \\
\hline Life Event Checklist (LEC) & - & $x$ \\
\hline Family Adaptability and Cohesion Evaluation Scales (FACES- $\square$ ) & - & $\mathrm{x}$ \\
\hline Handedness & - & $\mathrm{x}$ \\
\hline $\begin{array}{l}\text { The Schedule for Affective Disorders and Schizophrenia for School-Age Children } \\
\text { — Epidemiologic Version for the Adolescents (K-SADS-E) }\end{array}$ & - & $\mathrm{x}$ \\
\hline \multicolumn{3}{|l|}{ For parents } \\
\hline Child Behavior Checklist (CBCL) & - & $\mathrm{x}$ \\
\hline Centers for Epidemiological Studies — Depression scale (CES-D) & $\mathrm{x}$ & $\mathrm{x}$ \\
\hline Handedness & - & $\mathrm{x}$ \\
\hline Diagnostic Interview for Genetic Studies (DIGS) & - & $\mathrm{x}$ \\
\hline
\end{tabular}


serum insulin at 30, 60, 90, and 120 minutes. All blood samples were aliquoted and stored at $-70^{\circ} \mathrm{C}$ until use. The concentration of plasma glucose and lipid profiles (total cholesterol, HDL-C, and triglyceride) were measured using the fasting specimen by an autoanalyzer (Hitachi 7250 special; Hitachi, Tokyo, Japan). Serum insulin levels were determined by a microparticle enzyme immunoassay using the AxSYM system from Abbott Diagnostics (Abbott Laboratories, Dainabot Co. Ltd., Tokyo, Japan). Plasma levels of cortisol, leptin, and adiponectin were determined using fasting blood samples.

Anthropometric data such as height and weight were measured on a standing position using a standardized protocol. BMI is calculated as the weight in kilograms divided by the height in meters squared. After subjects had rested in a seated position for 10 minutes, systolic and diastolic blood pressure were measured automatically according to a common protocol by using the DINAMAP ${ }^{\mathrm{TM}}$ Vital Signs Monitor (Model 1846SX/P; Critikon Inc., Tampa, USA). Three separate readings were recorded 1 minute apart. The second and third measurements were averaged for data analysis.

\section{Future Works}

Preliminary analysis showed that there was substantial heritability for the metabolic function indexes and anxiety/depression in this healthy sample of adolescents. Furthermore, associations between anxiety/ depression and metabolic risk profile were observed. In a separate analysis, influences of genes on adiponectin were similar to those of shared environment for girls, whereas the influence of genes was slightly greater than those of shared environment for boys. This finding highlights possible shared environment avenues for early prevention or intervention of adiponectin-related metabolic abnormalities. Further studies examining the genetic and environmental contribution to the association of anxiety/depression with hormonal factors will be conducted.

\section{Acknowledgments}

We thank Mr. Fu-Shiung Lin for expert technical assistance with the biochemical assays, Ms. Chia-Lin Chao, Li-Fen Chien, Chien-Yi Chuang, and Chia-Yen Wu for excellent data collection, Mr. Po-Chang Hsiao for providing help in establishing database, and especially our participants for their support. The study was supported by National Health Research Institutes (NHRI-CNMG-9001S), National Science Council (NSC 93-2752-B-0020009-PAE, NSC 93-2320-B-002-069), and the National Taiwan University Center for Genomic Medicine.

\section{References}

Achenbach, T. M. (1991). Manual for the Child Behavior Checklist/4-18 and 1991 Profile. Burlington, VT: University of Vermont, Department of Psychiatry.
Atmaca, M., Tezcan, E., Kuloglu, M., \& Ustundag, B. (2005). Serum leptin levels in obsessive-compulsive disorder. Psychiatry and Clinical Neurosciences, 59, 189-193.

Bjorntorp, P. (2001). Do stress reactions cause abdominal obesity and comorbidities? Obesity Reviews, 2, 73-86.

Chen, C. J., Lin, T. M., Chang, C., \& Cheng, Y. J. (1987). Epidemiological characteristics of twinning rates in Taiwan. Acta Geneticae Medicae et Gemellologiae, 36, 335-342.

Chen, W. J., Chang, H. W., Wu, M .Z., Lin, C. C., Chang, C., Chiu, Y. N., \& Soong, W. T. (1999). Diagnosis of zygosity by questionnaire and polymarker polymerase chain reaction in young twins. Behavior Genetics, 29, 115-123.

Chen, W. J., Liu, S. K., Chang, C. -J., Lien, Y. -J., Chang, Y. -H., \& Hwu, H. -G. (1998). Sustained attention deficit and schizotypal personality features in nonpsychotic relatives of schizophrenic patients. American Journal of Psychiatry, 155, 1214-1220.

Cook, S., Weitzman, M., Auinger, P., Nguyen, M., \& Dietz, W. H. (2003). Prevalence of a metabolic syndrome phenotype in adolescents: Findings from the Third National Health and Nutrition Examination Survey, 1988-1994. Archives of Pediatrics and Adolescent Medicine, 157, 821-827.

Golden, S. H., Williams, J. E., Ford, D. E., Yeh, H. C., Paton Sanford, C., Nieto, F. J., \& Brancati, F. L. (2004). Depressive symptoms and the risk of type 2 diabetes: the Atherosclerosis Risk in Communities study. Diabetes Care, 27, 429-435.

Kraus, T., Haack, M., Schuld, A., Hinze-Selch, D., \& Pollmacher, T. (2001). Low leptin levels but normal body mass indices in patients with depression or schizophrenia. Neuroendocrinology, 73, 243-247.

Liuzzi, A., Savia, G., Tagliaferri, M., Lucantoni, R., Berselli, M. E., Petroni, M. L., De Medici, C., \& Viberti, G. C. (1999). Serum leptin concentration in moderate and severe obesity: relationship with clinical, anthropometric and metabolic factors. International Journal of Obesity and Related Metabolic Disorders, 23, 1066-1073.

Livshits, G., Pantsulaia, I., \& Gerber, L.M. (2005). Association of leptin levels with obesity and blood pressure: Possible common genetic variation [published erratum appears in the International Journal of Obesity and Related Metabolic Disorders, 2005, 29, 447]. International Journal of Obesity, 29, 85-92.

McCaffery, J. M., Niaura, R., Todaro, J. F., Swan, G. E., \& Carmelli, D. (2003). Depressive symptoms and metabolic risk in adult male twins enrolled in the National Heart, Lung, and Blood Institute twin study. Psychosomatic Medicine, 65, 490-497.

Musselman, D. L., Betan, E., Larsen, H., \& Phillips, L. S. (2003). Relationship of depression to diabetes types 1 
and 2: Epidemiology, biology, and treatment. Biological Psychiatry, 54, 317-329.

Nurnberger, J. I., Jr., Blehar, M. C., Kaufmann, C. A., York-Cooler, C., Simpson, S. G., Harkavy-Friedman, J., Severe, J. B., Malaspina, D., Reich, T., and collaborators from the NIMH Genetics Initiative (1994). Diagnostic Interview for Genetic Studies: Rationale, unique features, and training. Archives of General Psychiatry, 51, 849-859.

Puig-Antich, J., \& Orvaschel, H. (1990). Schedule for affective disorder and schizophrenia for school age children: Epidemiologic version and present episode version. Pittsburgh: Western Psychiatric Institute and Clinic.

Raikkonen, K., Matthews, K. A., \& Kuller, L. H. (2002). The relationship between psychological risk attributes and the metabolic syndrome in healthy women: antecedent or consequence? Metabolism: Clinical and Experimental, 51, 1573-1577.

Schmitz, S., Cherny, S. S., \& Fulker, D. W. (1998). Increase in power through multivariate analysis. Behavior Genetics, 28, 357-363.
Weiss, R., Dziura, J., Burgert, T. S., Tamborlane, W. V., Taksali, S. E., Yeckel, C. W., Allen, K., Lopes, M., Savoye, M., Morrison, J., Sherwin, R. S., \& Caprio, S. (2004). Obesity and the metabolic syndrome in children and adolescents. New England Journal of Medicine, 350, 2362-2374.

Winokur, A., Maislin, G., Phillips, J. L., \& Amsterdam, J. D. (1988). Insulin resistance after oral glucose tolerance testing in patients with major depression. American Journal of Psychiatry, 145, 325-330.

Yang, H. -J., Soong, W. -T., Chang, C. -N., \& Chen, W. J. (2000). Competence and behavioral/emotional problems among Taiwanese adolescents as reported by parents and teachers. Journal of the American Academy of Child and Adolescent Psychiatry, 39, 232-239.

Yang, H. -J., Soong, W. -T., Kuo, P. -H., Chang, H. -L., \& Chen, W. J. (2004). Using the CES-D in a two-phase survey for depressive disorders among nonreferred adolescents in Taipei: A stratum-specific likelihood ratio analysis. Journal of Affective Disorders, 82, 419-430. 